\title{
Annual Parallax Measurements of an Infrared Dark Cloud MSXDC G034.43+00.24
}

\section{Tomoharu Kurayama}

\author{
Department of Physics and Astronomy, Graduate School of Science and Engineering, \\ Kagoshima University, 1-21-35 Korimoto, Kagoshima 890-0065, Japan \\ email: kurayama@sci.kagoshima-u.ac.jp
}

\begin{abstract}
We have measured the annual parallax of the $\mathrm{H}_{2} \mathrm{O}$ maser associated with an infrared dark cloud, MSXDC G034.43+00.24, with VERA. The parallax is $0.643 \pm 0.049$ mas, corresponding to a distance of $1.56_{-0.11}^{+0.12} \mathrm{kpc}$. This value is less than half of the previous kinematic distance of $3.7 \mathrm{kpc}$. We revised the core-mass estimates of MSXDC G034.43+00.24 from the previous estimations of $1000 M_{\odot}$ to hundreds of $M_{\odot}$. The spectral type is still consistent with that of the massive star.
\end{abstract}

Keywords. astrometry, IRDC, star formation

\section{Introduction}

MSXDC G034.43+00.24 is one of the Infrared Dark Clouds (IRDCs), which are known as sites of massive star formation. Various parameters are derived for IRDCs (e.g., Sanhueza et al. (2010)). MSXDC G034.43+00.24 has four millimeter cores from $1.2 \mathrm{~mm}$ continuum observations (Rathborne et al. (2005)). An IRAS point source and a compact $\mathrm{H}$ II region are associated with the brightest millimeter core MM2. Three millimeter cores MM1, MM3 and MM4 have $\mathrm{H}_{2} \mathrm{O}$ maser sources revealed from VLA observations (Wang et al. (2006)). The kinematic distance of MSXDC G034.43+00.24 is $3.7 \mathrm{kpc}$ (Simon et al. (2006)), which is the only one distance estimation so far.

Various parameters, such as masses, luminosities and spectral types are deived from various observations (e.g., Sanhueza et al. (2010)), but many of them depend on the distance. However, some kinematic distances are far from the actual distances, derived from the annual parallaxes (e.g., Sato et al. (2010), Motogi et al. (2011)). It is not good to use kinematic distance for each source. Therefore, we have measured the annual parallax of this source with VERA.

\section{Observations, Results and Discussion}

Observations were carried out with VERA four stations. They were phase-referencing VLBI observations at $22 \mathrm{GHz}$ band. The phase-reference source is GPSR5 35.946+0.379 (= VCS2 J1855+0251), which is separated by 1.6 from MSXDC G034.43+00.24.

The results of position measurements are shown in Figure 1. We can trace the movements on the sky for five maser features in MM1. Declination data have large scatters caused by the strong sidelobes in the synthesized beam because the declination of this source $(\sim+1.5)$ is very close to $0^{\circ}$.

We carried out the least square fitting for the annual parallax, using the data points of right ascensions only. The resultant annual parallax is $0.643 \pm 0.049$ mas, which corresponds to the distance of $1.56_{-0.11}^{+0.12} \mathrm{kpc}$. This value is less than the half of the kinematic distance of $3.7 \mathrm{kpc}$. The details of this fitting is described in Kurayama et al. (2011). 

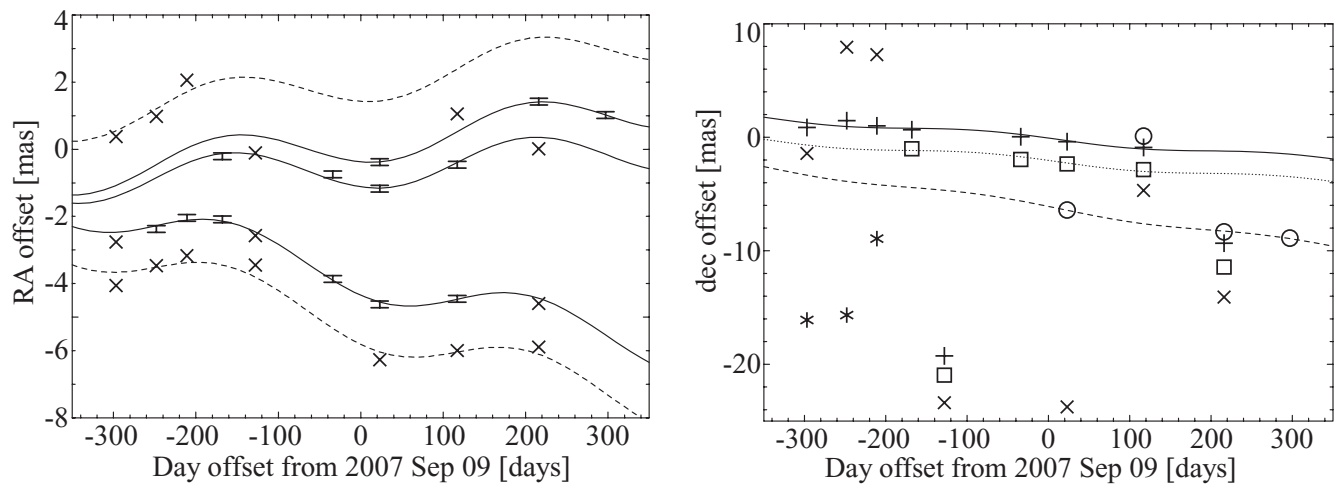

Figure 1. (Left) Plot of right ascensions versus time. Each line shows the different maser features. Crosses denotes the data which are not used for the least square fitting to derive the annual parallax. (Right) Plot of declinations versus time. Different signs denote different maser features. Lines are drawn from the least square fitting of the proper motion and the initial position by using the annual parallax of $0.643 \pm 0.049$ mas.

\begin{tabular}{crrrrrrrr}
\hline & \multicolumn{3}{c}{$\begin{array}{c}\text { Kinematic distance } \\
(3.7 \\
\end{array}$} & Mpc) & \multicolumn{4}{c}{ Distance from } \\
Millimeter core & MM2 & MM3 & MM4 & MM1 & MM2 & MM3 & MM4 \\
\hline Virial mass & 1130 & 1510 & 1370 & $\ldots$ & 476 & 637 & 578 & $\ldots$ \\
LTE mass & 330 & 1460 & $\ldots$ & $\ldots$ & 59 & 260 & $\ldots$ & $\ldots$ \\
Dust mass & 1187 & 1284 & 301 & 253 & 211 & 228 & 54 & 45 \\
\hline Bolometric luminosity & 32000 & $\ldots$ & 9000 & 12000 & 5700 & $\ldots$ & 1600 & 2100 \\
Spectral type & O9.5 & $\ldots$ & B0.5 & B0.5 & B1 & $\ldots$ & B3 & B2 \\
\hline
\end{tabular}

Table 1. Modification of physical parameters of millimeter cores in the infrared dark cloud, MSXDC G034.43+00.24. The units of masses and luminosities are solar masses and solar luminosities. Data from Sanhueza et al. (2010), Rathborne, Jackson \& Simon (2006), and Rathborne et al. (2005).

Distance is very fundamental parameter, so variours parameters have changed by the change of distances. Table 1 shows examples of this change. Masses are reduced from $\sim 1000 M_{\odot}$ to hundreds $M_{\odot}$. Spectral types still shows that they will be massive stars.

\section{References}

Kurayama, T., Nakagawa, A., Sawada-Satoh, S., Sato, K., Honma, M., Sunada, K., Hirota, T., \& Imai, H. 2011, PASJ, 63, 513

Motogi, K., Sorai, K., Habe, A., Honma, M., Kobayashi, H., \& Sato, K. 2011, PASJ, 62, 101

Rathborne, J. M., Jackson, J. M., \& Simon, R. 2006, ApJ, 641, 389

Rathborne, J. M., Jackson, J. M., Chambers, E. T., Simon, R., Shipman, R., \& Frieswijk, W. 2005, ApJ, 630, L181

Sanhueza, P., Garay, G., Bronfman, L., Mardones, D., May, J., \& Saito, M. 2010, ApJ, 715, 18

Sato, M., Hirota, T., Reid, M. J., Honma, M., Kobayashi, H., Iwadate, K., Miyaji, T., \& Shibata, K. M. 2010, PASJ, 62, 287

Simon, R., Rathborne, J. M., Shah, R. Y., Jackson, J. M., \& Chambers, E. T. 2006, ApJ, 653, 1325

Wang, Y., Zhang, Q., Rathborne, J. M., Jackson, J., \& Wu, Y. 2006, ApJ, 651, L125 